(Aus der Universitäts-Augenklinik zu Würzburg.)

\title{
Experimenteller Beitrag zur Frage nach dem Sitz and Wesen der Accommodationsparese bei bakteriellen Intoxikationskrankheiten.
}

\section{Die Accommodationsparese bei Botulismus.}

Von

Dr. Paul Römer, Privatdozenten, und Dr. Ludwig Stein, Assistenten der Klinik.

Die im folgenden mitzuteilenden Untersuchungen haben den Zweck, auf einem bisher in der Ophthalmologie nicht beschrittenen experimentellen Wege weitere Aufklärung über den Sitz und das Wesen der Accommodationsparesen im Verlaufe der bakteriellen Intoxikationskrankheiten zu erlangen.

Trotzdem seit geraumer Zeit bekannt ist, dass die Krankheitserscheinungen bei solchen Infektionskrankheiten, die häufig eine Accommodationsparese im Gefolge haben, auf der Wirkung ganz bestimmter Toxine beruhen, deren Einfluss auf den tierischen Organismus wir jetzt studieren können, ist unseres Wissens von ophthalmologischer Seite bisher noch nicht der Versuch gemacht worden, an der Hand des Toxinproblemes experimentell den Sitz der Accommodationsparese bei Krankheiten wie Diphtherie und Botulismus zu erforschen.

Und doch kann es unseres Erachtens keinem $Z$ weifel unterliegen, dass dieser $W$ eg in erster Linie beschritten werden muss, wenn wir zu richtigen Anschauungen über das Wesen und den Sitz dieser in praxi so häufig vorkommenden Accommodationsparesen gelangen wollen.

Denn einwandfreie menschliche Präparate von Accommodationsparese bei Diphtherie und Botulismus zur anatomischen Untersuchung zu bekommen, wird stets mit den grössten Schwierigkeiten verknüpft sein, da es sich, wie aus unsern Untersuchungen hervorgehen wird, hier um feinste Veränderungen handelt. 
Aber abgesehen davon, dass die Anwendung der Toxinlehre berufen sein wird, uns definitive Aufklärung über den Sitz dieser Accommodationsparesen zu bringen, ist sie weiter schon jetzt im stande, uns für wichtige klinische Erscheinungen dieser Krankheitsbilder die bisher fehlende Erklärung zu geben.

Wenn wir fragen, welche klinischen Erscheinungen der Accomroodationsparesen einer Erklärung noch bedürfen, so sind es folgende:

Wie ist es zu verstehen, dass die Accommodationsparese bei Diphtherie eine so lange Inkubation besitzt, und wie ist es zu erklären, dass gerade diese Lähmungen nach Massgabe der klinischen Erfahrung durch das Diphtherieantitoxin so wenig oder gar nicht beeinflusst werden?

Auf beide Fragen ist eine befriedigende Antwort bisher nicht gegeben worden.

Nach der Entdeckung des Diphtheriegiftes mussten wir uns mit der Annahme begnügen, dass die Toxine der Diphtheriebacillen hier eine Rolle spielen. Deshalb schrieb Schirmer: "Vielmehr können nach dem heutigen Stand unserer Kenntnisse nur die Stoffwechselprodukte des Bacillus, die Toxine in Frage kommen", und er glaubte, dass diese letzteren in besonders wenig widerstandsfähigen Geweben Entzündung hervorrufen.

Schirmer sucht zwar den nach seinem Hinweis auf die Toxine sich selbst gemachten Einwand: „Frappierend erscheint bei der eben skizzierten Hypothese, dass die Lähmungen erst einige Zeit nach Ablauf der Diphtherie eintreten, während doch wabrscheinlich die Konzentration der Toxine während der Krankheit am grössten ist", durch folgende Bemerkung zu entkräften: „Dem ist aber entgegenzuhalten, dass anatomisch der Beginn der Neuritis in der Tat verschiedentlich nachgewiesen werden konnte bei Patienten, die mit florider Diphtherie gestorben waren, dass die Entzündung des Endoneuriums eine bestimmte Höhe erreichen muss, ehe sie die wahrscheinlich sekundären Veränderungen in den Nervenfasern selbst induzieren kann, und dass wir bisher nicht wissen, wie schnell der Organismus die Toxine wieder ausscheidet."

Es bedarf kaum eines Hinweises, dass ganz abgesehen zunächst von jeder Erörterung des Sitzes der Accommodationsparese bei Diphtherie und ihrer anatomischen Grundlage diese Ausführungen Schirmers noch keine genügende Erklärung für die lange Inkubationszeit der Accommodationsparese abgeben. Denn es ist selbstverständlich, dass wir aus anatomischen Befunden bei florider Diphtherie, bei 
Exper. Beitrag z. Frage nach d. Sitz u. Wesen d. Accommodationsparese usw. I. 293

denen in den meisten Fällen nicht einmal $\mathrm{zu}$ entscheiden ist, was Diphtheriegiftwirkung, was sekundäre septische Veränderungen sind, nicht ohne weiteres Schlüsse auf irgendwelche Befunde bei Accommodationsparesen ziehen dürfen, die in der Rekonvalescenz auftreten. Wir werden noch sehen, dass wir gerade bei der Diphtherieintoxikation nicht die akute der chronischen vergleichen dürfen.

Was die zweite wesentliche Erscheinung in der Pathogenese der Accommodationsparese angeht, dass dieselbe vom Diphtherieantitoxin nicht beeinflusst wird, so hat Schirmer keine Erklärung für dieselbe versucht. Er erwähnt nur, dass nach der damaligen Diskussion das Toxin nicht auf chemischem Wege vom Antitoxin paralysiert werde.

Auch hierin hat sich seit jener Zeit viel geändert. Schon ein Jahr nach der Schirmerschen Abhandlung ist von Ehrlich durch geniale Versuche der einwandfreie Beweis erbracht, dass sich Toxin und Antitoxin auf keinem andern als auf chemischem Wege paralysieren.

Die Frage kann daher in der Pathogenese der Accommodationsparese heute nur noch lauten: Wie kommt es, dass trotzdem das Toxin rom Antitoxin chemisch paralysiert wird, die Accommodationsparese vom Heilserum nicht beeinflusst wird?

Auf Grund der Ergebnisse der Immunitätsforschung sind wir zum ersten Male im stande, eine hinreichende Erklärung zu geben sowohl für das Wesen der langen Inkubationszeit, als auch für die Tatsache, dass diese Lähmungen nicht von dem Diphtherieantitoxin beeinflusst werden.

Zum Verständnis ist es notwendig, dass wir kurz die Neutralisationsverhältmisse des Diphtheriegiftes betrachten. Auf Einzelheiten freilich einzugehen müssen wir uns versagen. Es muss in dieser Hinsicht auf die Werke der Immunitätsforschung verwiesen werden.

Das Diphtheriegift ohne Antitoxinzusatz tötet bekanntlich in bestimmten Dosen Meerschweinchen in wenigen Tagen unter ganz charakteristischen klinischen und anatomischen Erscheinungen. Sobald man jedoch zu einer bestimmten Diphtheriegiftmenge, am besten der Ehrlichschen LO-Dosis, die ein Multiplum der tödlichen Minimaldosis enthält, und von einer Immunitätseinheit gerade neutralisiert wird, nur Bruchteile einer I. E. hinzufügt, bekommt man bei geeigneten Verhältnissen beider Substanzen Gift-Antitoxingemische in die Hand, welche ganz andere Eigenschaften am tierischen Organismus entfalten, als das ungesättigte Gift. Während das Diphtheriegift ausgedehnte Infiltrationen mit Hautnekrosen und Haarausfall hervorruft und akut zum Tode führt, haben jene partiell mit Anti- 
toxin abgesättigten Toxine die Fähigkeit, akut tödlich zu wirken, verloren, machen nur noch kleine, ganz weiche Infiltrationen, und rufen nach einer bestimmten, mehrere Wochen dauernden Inkubationsperiode eigentümliche Lähmungen bei den Tieren hervor.

Diese fundamentale Tatsache aus dem Gebiet der Neutralisationsvorgänge zwischen Diphtherietoxin und Antitoxin hat Ehrlich durch den mittels seiner Methode der partiellen Absättigung erbrachten Nachweis erklärt, dass das Diphtherietoxin keine einheitliche Substanz darstellt, sondern zwei verschiedene Sekretionsprodukte des Diphtheriebacillus enthält: das Toxin und das Toxon.

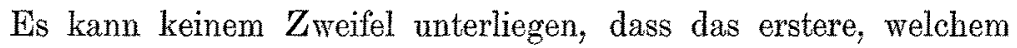
die akute Wirkung zukommt, eine starke Avidität zum Antikörper besitzt und darum in Gift-Antitoxinlösungen zunächst vom Antitoxin gebunden wird.

Die Toxone dagegen haben eine viel geringere Affinität zum Antikörper, und sie sind es, welche die Paresen im tierischen Organismus auslösen.

Aus allen Untersuchungen über die Toxone des Diphtheriebacillus, die hauptsächlich von Ehrlich und Madsen ausgeführt sind, geht hervor, dass Produlkte des Diphtheriebacillus unter geeigneten Bedingungen mehrere Wochen nach ihrer Einführung in den tierischen Organismus bestimmte Lähmungen hervorrufen können.

Diese experimentelle Tatsache ist geeignet, auf die Frage nach der Entstehung der diphtheritischen Lähmung beim Menschen ein neues Licht zu werfen. Auch beim Menschen erscheinen die Lähmungen, und speziell die uns hier interessierenden Accommodationsparesen, erst nach einer langen Inkubationsdauer. Wenn es daher für den tierischen Organismus Produkte des Diphtheriebacillus gibt, mit denen wir experimentell charakteristische Lähmungen hervorrufen können, so werden wir annehmen dürfen, dass durch dieselben auch beim Menschen derartige Prozesse nach ähnlicher Zeitdauer ausgelöst werden.

Die Erfahrungen der Immunitätsforschung haben, wie ich aus der liebenswürdigen persönlichen Mitteilung von Herrn Geheimrat Ehrlich weiss, ergeben, dass der Gehalt der Diphtheriegifte an Toxonen grossen Schwankungen unterworfen ist.

Es ist daher durchaus begreiflich, dass nicht jede Diphtherieinfektion von einer Lähmung gefolgt zu werden braucht. Dazu kommt, 
Exper. Beitrag z. Frage nach d. Sitz u. Wesen d. Accommodationsparese usw. I. 295

dass mannigfache Einflüsse der Behandlung, rechtzeitige oder späte Seruminjektion von Einfluss sein müssen.

Und gerade die experimentellen Erfahrungen über die Grenzen der Wirkung des Diphtherieheilserums gegenüber den Toxonen des Diphtheriegiftes sind geeignet, unsere Anschaunng, dass auch die Accommodationsparese des Menschen bei Diphtherie eine Toxonwirkung ist, zu stiitzen. Wir verdanken diese Untersuchungen ror allem Madsen und seinem Schüler Dreyer.

Es war durch die Untersuchungen von Dönitz bekannt, dass bei intravenöser Injektion das Diphtheriegift bei Tieren ausserordentlich schnell von den Geweben gebunden wird. Bei siebenfach tödlicher Minimaldosis konnte Dönitz schon 15 Minuten nach der Gifteinspritzung die Tiere nicht mehr mit der ebenfalls intravenös verabreichten in vitro gerade neutralisierenden Antitoxinmenge retten.

Entsprechend der geringen Affinität der Toxone dagegen war zu erwarten, dass dieselben langsamer im Innern des Organismus an die empfänglichen Zellen gebunden würden. Dreyer injizierte zur Prüfung dieser Frage Kaninchen intravenös von einem Gifte $0,5 \mathrm{ccm}+{ }^{175 / 200}$ I. E., eine Mischung, in der 25 Toxonäquivalente frei waren und welche nach einer Inkubationszeit von 12-18 Tagen eine letale Parese hervorrief. Nach verschiedenen Zeitabschnitten wurde dann ebenfalls intravenös die in vitro neutralisierende Dosis Antitoxin ${ }^{20} / 200$ T. E. nachgeschickt. Da diese Versuche für unsere Frage prinzipielles Interesse haben, füge ich eine der Tabellen von Dreyer hier ein:

\begin{tabular}{|c|c|c|c|c|c|c|c|c|}
\hline \multirow{2}{*}{$\begin{array}{c}\text { Gewicht } \\
\text { des Versuchs- } \\
\text { tieres in } \bar{F}\end{array}$} & \multirow{2}{*}{$\begin{array}{l}\text { Anzahl } \\
\text { der } \\
\text { freien } \\
\text { Toxon- } \\
\text { tquiva- } \\
\text { lente }\end{array}$} & \multicolumn{2}{|c|}{ Antitoxin } & \multicolumn{4}{|c|}{ Paresen } & \multirow{2}{*}{$\begin{array}{c}{ }^{\dagger} \\
\text { Tage } \\
\text { nach der } \\
\text { In- } \\
\text { jektion }\end{array}$} \\
\hline & & $\begin{array}{l}\text { Minuten oder } \\
\text { Stunden } \\
\text { nach der } \\
\text { Injeltion }\end{array}$ & $\begin{array}{c}\text { Menge } \\
\text { in } \\
\text { I. E. }\end{array}$ & $\begin{array}{c}\text { Inku- } \\
\text { bations- } \\
\text { zeit } \\
\text { in Tagen }\end{array}$ & Graa & $\begin{array}{c}\text { Dauter } \\
\text { in } \\
\text { Tagen }\end{array}$ & Verlauf & \\
\hline 1240 & 25 & 1 Minut. & $25 \%$ & 0 & 0 & 0 & 0 & lebt \\
\hline 1310 & 25 & 2 Std. & $"$ & 0 & 0 & 0 & 0 & $"$ \\
\hline 1290 & 25 & 2 & $"$ & 0 & 0 & 0 & 0 & ", \\
\hline 1320 & 25 & & $"$ & 0 & 0 & 0 & 0 & $"$ \\
\hline 1500 & 25 & 5 & $"$ & 27 & leicht & 12 & geheilt & $"$ \\
\hline 1335 & 25 & 10 & $"$ & 22 & mittelstark & 15 & 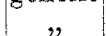 & $"$ \\
\hline 1250 & 25 & 10 & $"$ & 20 & & 19 & & \\
\hline 1470 & 25 & 16 & 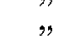 & 14 & stark & 13 & $\stackrel{3}{+}$ & 17 \\
\hline 1400 & 25 & 16 & " & 0 & 0 & 0 & , & $\left.16^{x}\right)$ \\
\hline 1400 & 25 & 24 & $"$ & 13 & stark & 4 & & 17 \\
\hline 1400 & 25 & 24 & , & 13 & mittelstark & 31 & geheilt & lebt \\
\hline $13401 \mathrm{Kon}-$ & 25 & 0 & $"$ & 13 & stark & 3 & + & 16 \\
\hline 1210 f trollen & 25 & 0 & $"$ & 13 & $"$ & 4 & $\dagger$ & 16 \\
\hline
\end{tabular}

1) An eitriger Pericarditis. 
Aus der Tabelle ist zu ersehen, dass im Gegensatz zu der Diphtherietoxinwirkung die Toxonwirkung noch nach zwei Stunden unschädlich gemacht werden konnte. Und auch bei einem Intervall von zehn Stunden war noch eine antitoxische Wirkung vorhanden. Waren dagegen 24 Stunden nach der Toxoninjektion verstrichen, so konnte die in vitro neutralisierende Antitoxinmenge die Paresen nicht mehr verhindern. Derartige Versuche liefern die experimentelle. Erklärung für die Tatsache, dass beim Menschen die diphtheritischen Lähmungen und so auch die Accommodationsparesen so wenig vom Antitoxin beeinflusst werden. Die Toxone haben an sich schon eine geringe Affinität zum Antitoxin. Ist gar die Verankerung der haptophoren Gruppen der Toxone an die giftempindlichen Zellen erfolgt, so sind die Antitoxine nicht mehr im stande, diese Verbindung zu sprengen, diese Körper den Geweben zu entziehen, die Lähmung zu verhüten oder zu heilen.

Es liegen, wie wir noch sehen werden, bereits experimentelle Beweise vor, dass die Verhältnisse der mangelhaften Antitoxinbeeinflussung auch beim Botulismus ganz ähnlich sind wie bei der Diphtherie. Der Unterschied liegt beim Botulismus nur in der Kürze der Inkubationsperiode. Aber dies kann uns nicht wundernehmen. Die einzelnen bakteriellen Toxine haben je nach ihrem Resorptionskoeffizienten, je nach der Stärke der Affinität zu bestimmten Zellen. eine verschieden lange Inkubationszeit. Das wesentlichste Moment jedoch für das Verständnis des Wesens der Accommodationsparesen ist auch hier die Tatsache, dass gleich allen andern bakteriellen Toxinen auch das Botulismustoxin, wie man sich im Experiment leicht überzeugen kann, eine charakteristische Inkubationsperiode besitzt. Man kann Tieren die grösste Menge Botulismustoxin einführen, das Mindestintervall für das Auftreten der ersten Erscheinungen kann durch keine Vergrösserung der Giftdosis aufgehoben werden. Durch diese Eigenschaft, sowie durch die Fähigkeit der Antitoxinerzengung unterscheiden sich ja alle bakteriellen Toxine bekanntlich von den chemisch definierten Giften. Und dies sind, abgesehen von den klinischen Merkmalen, die bakteriologischen Gründe, welche uns berechtigen, das Wesen der Inkubationsdauer und der mangelhaften Beeinflussung durch die Antitoxine bei beiden Arten von Accommodationsparesen von einheitlichem Gesichtspunkt zu betrachten.

Nach diesen Ausführungen, welche lediglich den Zweck hatten, eine auf der Grundlage der Immunitätsforschung ruhende Erklärung für die wichtigsten bisher unerklärlichen Erscheinungen des klinischen 
Bildes dieser Paresen zu geben, und damit eine Lücke unserer Kenntnisse über diese Krankheitsbilder auszufüllen, gehen wir jetzt zu den Untersuchungen über den Sitz dieser Affektionen über. Wir durften diese Erklärung der Mitteilung unserer anatomischen Untersuchungen vorausschicken, denn diese Erklärung der Inkubationszeit und des Mangels antitoxischer Beeinflussung bleibt bestehen, ganz gleichgiiltig, ob die Toxinwirkung sich am peripheren Nerven, am Muskel oder am Zentralnervensystem abspielt, ob sich dieselbe in Gestalt einer Entzündung oder Degeneration usw. äussert. Denn überall handelt es sich bei der Toxinwirkung um chemische Affinitäten zwischen einzelnen Bestandteilen der Zellen, um feste Verankerungen haptophorer Gruppen an bestimmte Rezeptoren der Zellen, die bezïglich ihrer Sprengung und Beeinflussung durch Antikörper den Neutralisationsgesetzen der Antitoxine unterworfen sind.

Wir begannen unsere Studien mit dem Botulismustoxin, weil uns aus andern und eigenen Untersuchungen bekannt war, dass bei der Botulismusvergiftung der Tiere Störungen von seiten der Pupillen hervorgerufen werden können, und weil wir auf diese $W$ eise von seiten der Augen eine genügende Kontrolle haben, ob etwaige für die anatomische Untersuchung hinreichende Veränderungen zu erwarten sind, während wir bezüglich einer Accommodationsparese bei kranken Tieren meistens auf Vermutungen angewiesen sind. Wenigstens wird es bei schwerkranken Tieren nur mit Schwierigkeit gelingen, genügende Anhaltspunkte für das Vorhandensein einer derartigen Parese zu gewinnen, abgesehen davon, dass z. B. Kaninchen, Hunde usw. nur ein sehr geringes Accommodationsvermögen besitzen.

Unter Botulismus werden in der ophthalmologischen Literatur noch die heterogensten Dinge zusammengeworfen. Muscheln-, Austern-, Fisch-, Fleischvergiftungen werden, weil sie zum Teil analoge Krankheitsbilder hervorrufen, unter einheitlichen Gesichtspunkten zusammengefasst. Es ist notwendig, zu betonen, dass hier mehr als dies bisher geschehen ist, ätiologisch zu trennen sein wird.

Seitdem van Ermengem den Erreger des Botulismus entdeckt hat, ist es selbstverständlich, dass wir, streng genommen, als Botulismus nur solche Krankheitsbilder bezeichnen dürfen, deren ätiologische Natur im Sinne jenes Forschers erwiesen ist. Und dies ist von ophthalmologischer Seite noch nicht genügend geschehen.

Nur der eine von uns (Römer) war in der Lage, als erster die Untersuchungen van Ermengems kontrollieren und bestätigen zu können. Es gelang bei einer Schinkenvergiftung in einer Familie, 
von der das eine Mitglied Accommodationsbeschwerden bekommen hatte, den Bacillus botulinus zu züchten und seine Identität mit dem van Ermengems festzustellen.

Der Bacillus selbst ist strenger Anaerobier und Parasit, der sich im lebenden Körper nicht vermehrt, dagegen in den toten Substraten enorm giftige Toxine produziert, die an Giftigkeit nur mit dem Tetanustoxin verglichen werden können.

Zur Kenntnis der klinischen Symptome bei der Botulismusvergiftung sei folgendes bemerkt. Schon bei seinen frühern Versuchen fiel es Römer auf, dass diese Toxine beim Lagern der Bouillon in ihrem toxischen Titre eine Zeitlang heruntergingen. Diese Beobachtungen der Giftabschwächung beim Botulismustoxin, die damals keine weitere Beachtung fanden, haben deshalb jetzt wieder ein erhöhtes Interesse, seitdem Ehrlich gezeigt hat, dass bei diesem Lagern bakterieller Toxine ein teilweiser Zerfall der toxophoren Gruppen eintritt und so eine Umwandlung der Toxine in Toxoide erfolgt. Wir erwähnen diese Beobachtungen über die Abschwächung des Botulismustoxins nur deshalb, weil sie von künftigen Nachuntersuchern beachtet werden müssen, und weil sie uns verstehen lassen, warum bei ein und derselben Spezies, je nachdem man frische oder abgelagerte Gifte anwendet, die Krankheitssymptome anch von seiten der Augen nicht unerheblich differieren können.

So bewirkte das früher von $\mathrm{Römer}$ dargestellte Toxin in frischem Zustand bei Meerschweinchen eine absolute Starre der Pupillen, während die Verwendung alter abgelagerter Gifte nur eine Erweiterung mit allerdings sehr träger Reaktion zur Folge hatte. Auch ist zu beachten, dass immer frische Verdünnungen des Giftes aus der Stammlösung hergestellt werden müssen, weil auch das Botulismustoxin nach unsern Erfahrungen ähnlich dem Tetanustoxin in verdünnten Lösungen sich leicht auch bei strengster, stets kontrollierter Asepsis zersetzt.

Alle diese Momente aus der experimentellen Beschäftigung mit den Toxinen verdienen Beachtung, weil sie uns die Differenzen im klinischen Bild des Botulismus beim Menschen verständlich machen. Abgesehen von der Menge des zur Resorption gelangten Toxins wird es von solchen Schwankungen der ja sehr labilen Toxinkonstitution abhängen, $o b$ einzelne Muskelaktionen betroffen sind oder nicht, ob die Lähmung der Pupille stärker ist.als die der Accommodation, ob und in welchem Grade ausser der Binnenmuskulatur noch andere Muskeln betroffen sind.

Die Symptome der akuten Botulismusvergiftung hat van 
Ermengem bereits ausführlich geschildert. Sie waren bei der grossen Zahl der damals gleichzeitig Erkrankten kurz folgende:

Etwa 20-36 Stunden nach der Mahlzeit traten nach mehr oder weniger ausgesprochenen Magen-Darmbeschwerden die Störungen von seiten der Augen auf. Die Pupillen waren stark erweitert und reaktionslos. Dazu kam in den schweren Fällen Ptosis und Doppeltsehen. Die Kranken klagten ferner über brennenden Durst und hatten das Gefühl, als würden sie am Halse gewürgt. Die Pharynxschleimhaut war gerötet, und es bestanden starke Schlingbeschwerden, so dass feste Speisen gar nicht, flüssige nur mühsam genossen werden konnten. Der Heilungsverlauf war immer ein protrahierter, die Accommodationsparesen bestanden zum Teil monatelang. Von den $30 \mathrm{~Pa}-$ tienten starben drei, weitere zehn schwebten lange Zeit in Todesgefahr.

Analoge Symptomenkomplexe, wie sie beim Menschen hervortraten, hat van Ermengem dann mit den Toxinen des Bacillus botulinus an Tieren hervorrufen können. Unsere frühern und jetzigen Beobachtungen stimmen mit denen van Ermengems vollkommen überein.

Mäuse erkranken bei der ejnfach letalen Dosis sowohl nach Fütterung, wie subcutaner Injektion schon im Laufe von 24 Stunden. Sie sitzen mit gesträubten Haaren, verklebten Augen zusammengekauert. Werden sie zu Bewegungen gezwungen, kriechen sie oft, die Hinterfüsse nachziehend, auf dem Bauche hin, ohne im Gegensatz zu Tetanus Krampferscheinungen zu zeigen.

Emptänglich sind ferner Meerschweinchen, Ratten, Kaninchen und Katzen.

Vor allem interessiert uns aber der Krankheitsverlauf beim Affen. Aus van Ermengems Untersuchungen geht folgendes hervor. Nach Fütterung mit dem toxinhaltigen Extrakt aus dem betreffenden Schinken starben zwei Affen nach wenigen Stunden. Bei einem andern, welcher die kleinste Dosis bekommen hatte, traten schon nach 12 Stunden die ersten Erscheinungen auf. Das Tier wurde unruhig, die Augen wurden eigentümlich glänzend und tränten. Die Pupillen waren anfangs normal, später erweiterten sie sich, reagierten nur noch träge auf Licht und vergrösserten sich trotz Andauer des Reizes wieder. In der Nase und im Mund sammelte sich Schleim und verursachte Erstickungsanfälle. Nach einigen weitern Stunden nahmen die Erscheinungen rapide bis zum Tode zu. Das Tier kauerte sich zusammen, die Atmung wurde unregelmässig, mühsam, der Gesichtsausdruck starr, „die Augen, mit aussergewöhnlich erweiterten Pupillen, sind halb bedeckt durch die bewegungslosen Augenlider". 
In unsern eigenen Versuchen an Afien waren wir bestrebt, die Vergiftung möglichst so zu dosieren, dass die Erscheinungen nicht so akut verliefen. Wir glauben dies dadurch erreicht zu haben, dass wir zunächst genaue Toxizitätsbestimmungen an Mäusen ausführten. Es standen uns drei verschiedene Toxine zur Verfügung. Das eine hatte Herr Prof. Geppert in Giessen aus Kulturen ausgefällt, die früher von Römer angelegt waren. Das andere hatte uns Herr Prof. van Ermengem, das dritte Rerr Geheimrat Ehrlich zur Verfügung gestellt. Wir sprechen hiermit sämtlichen Herren unsern herzlichsten Dank aus.

Nach grössern Versuchsreihen an Mäusen entschieden wir uns für das schwächere, in Frankfurt hergestellte flüssige Toxin.

Bevor wir jedoch unsere Befunde an den Affen mitteilen, müssen wir noch kurz die pathologisch anatomischen Veränderungen besprechen, die bisher über die Botulismustoxinwirkung vorliegen. Denn diese Mitteilungen in der Literatur waren für uns mit Veranlassung gewesen, vom ophthalmologischen Gesichtspunkte aus dem Sitz der Augenveränderungen nachzugehen. Wir können hierbei von den Befunden an den übrigen Körperorganen absehen, weil dieselben für unsere Frage ron keinem Interesse sind, und uns auf die Veränderungen beschränken, die am Zentralnervensystem beobachtet worden sind. Die Stelle in der Arbeit des Entdeckers des Bacillus botulinus, welche sich hierauf bezieht, ist folgende:

„Beim Zentralnervensystem sind die Läsionen in dem Grosshirn fast Null; sie fehlen in den Nervenfasern und sind sehr ausgesprochen im Rückenmark, weniger in der Medulla oblongata, Protuberanz und Gehirnschenkel. Im Rückenimark und Pons sind sie fast ausschliesslich in den Vorderhörnern und Hinterhörnern (motorische Kerne der Glieder und Bulbärkerne) vorhanden. Im ersten Stadium findet man nur in den Nervenzellen eine von der Peripherie ausgehende Verminderung der ehromatophilen Elemente, später sind die Nisslsehen Körperehen in feine pulverartige Granula umgewandelt. Noch später entstehen Vakuolen durch Auflösung der achromatischen Substanz. Diese chromatolytischen Degenerationserscheinungen sind im Kern der N. Hypoylossus, im Nucleus ambiguus, im Nucleus dorsalis des N. vagus, in den Purkinjeschen Zellen des Kleinhirnes und im Mittelkern des Oculomotorius beobachtet worden."

Die feineren Veränderungen an den Ganglienzellen hat Marinesco für van Ermengem mit Hilfe der Nisslschen Methode untersucht.

Gleichzeitig und unabhängig von Marinesco haben Kempner und Pollack anatomische Untersuchungen über die Wirkung des 
Botulismustoxines auf die Ganglienzellen der Vorderhörner des Rücken markes bei Meerschweinchen, Kaninchen und Katzen angestellt. Die von den beiden Autoren erhobenen Befunde bei akuter Botulismusvergiftung stimmen im wesentlichen mit den von Marinesco beschriebenen überein. Nur im ersten Stadium sahen Kempner und Pollack dem Verschwinden der Nisslschen Körperchen eine klumpige Schwellung vorausgehen. Ferner konnten sie sich von einer Neurogliavermehrung nicht recht überzeugen. Die Hauptreränderungen an den motorischen Ganglienzellen im Rückenmark waren ebenso vorhanden, wie sie von Marinesco beobachtet waren. Es wird auch von Kempner und Pollack der Zerfall der Nisslschen Körperchen, das staubförmige Aussehen der Zellen geschildert. Bei chronisch vergifteten Katzen, die trotz klinischer schwerer Erscheinungen mit starken Paresen am Leben blieben, glaubten Kempner und Pollack mit der Nisslschen Methode noch nach Monaten homogene Trübung und Auflösung des Zellinhaltes konstatieren zu können. Von besonderem Interesse waren die Versuche von Kempner und Pollack, in denen entschieden werden sollte, ob das von Kempner im Institut für Infektionskrankheiten in Berlin hergestellte spezifische Antitoxin im stande war, die Zellen vor dem Toxin zu schützen und bereits erkrankte Zellen zu heilen.

Wenn man ihre Versuchsreihen uiberblickt, so ergibt sich, dass die Zeitdauer, in welcher das dem Gift nachgeschickte Antitoxin noch im stande war, die Zellen vor jeder Giftwirkung zu schützen, eine recht kurze war. Schon von der 20. Stunde nach der Intoxikation ab wiesen die Ganglienzellen schwere Veränderungen auf, auch wenn es mittels des Serums gelang, die Tiere am Leben zu erhalten. Diese experimentellen Resultate beweisen für unsere Frage jedenfalls, dass, auch wenn es gelingt, die zum Tode führende Intoxikation der lebenswichtigen Organe mittels antitoxischen Serums aufzuhalten, gleichwohl schon Zerfallserscheinungen an gewissen Ganglienzellen vorhanden sein können, die vom Serum nicht mehr beeinflusst werden können. In ganz analoger Weise werden die Verhältnisse beim menschlichen Botulismus liegen in den Fällen, wo die Vergiftung nicht zum Tode führt. Auch wenn der Organismus selbst nicht erliegt, werden charakteristische Veränderungen in bestimmten Zentren vorhanden sein können, die, wie z. B. die Dauer der Paresen zeigt, lange Zeit zu ihrer Restitution erfordern. Dass diese Veränderungen im Zentralnervensystem, die beim Botulismus unter anderem zur Accommodationsparese führen, etwa durch Hämorrhagien bedingt sein 
könnten, muss von vornherein unwahrscheinlich erscheinen. Denn es wäre nicht zu verstehen, wie solche Blutungen immer nur solche Veränderungen herbeifuihren könnten, die mit typischer Regelmässigkeit entstehen und wieder vergehen.

Vielmehr geht aus allen diesen bisherigen anatomischen Untersuchungen übereinstimmend hervor, dass das Botulismustoxin eine ausgesprochene Affinität zu bestimmten Zellkomplexen des Zentralnervensystems besitzt. Es war danach zu erwarten, dass sich dieses Toxin in erster Linie dazu eignen würde, auf dem Weg des Experimentes in die uns hier interessierende Frage nach dem Sitz der Accommodationsparese einige Klarheit $\mathrm{zu}$ bringen.

Zur Einübung der Technik untersuchten wir zunächst das Okulomotoriuskerngebiet von normalen Kaninchen, ferner von solchen, bei denen die Exenteration nnd Enucleation eines Auges einige Wochen vorher ausgeführt war.

Die Präparate wurden folgendermassen behandelt. Nach Dekapitation und Aufsägung des Schädels kam das betreffende Gehirnstïck in $96 \%$ Alkohol. Derselbe wurde am nächsten Tag erneuert und gleichzeitig wurde die Schnittfläche des Stïckes in der von Bernheimer angegebenen Weise zurechtgeschnitten. Nach eintägigem weitern Aufenthalt in absolutem Alkohol erfolgte die Einbettung in Paraffin. Die Schnitte hatten eine Dicke von $10 \mu$.

Was die Färbung angeht, so haben wir in den Vorversuchen sowohl wie in den Hauptversuehen die Überzeugung gewonnen, dass wir mit der Thionin- oder Thionin-Erythrosin-Färbung nach Einbettung in Paraffin ebenso gute Resultate bekommen haben, als mit der ursprünglichen Nisslschen Methode ohne vorausgegangene Einbettung. Mehr wie ${ }^{3 / 4}$ aller Schnitte sind genau nach Nissl nach vorausgeschiekter Einbettung gefärbt.

Infolgedessen haben wir uns an die einfachen Methoden nach Einbettung gehalten. Wir konnten dies um so mehr, als alle Präparate sowohl des Kontrolltieres als der Versuchstiere eine gleichmässige Behandlung: durchmachten.

Technisch sowohl wie in der Deutung der Bilder sind unsere Präparate von Herrn Prof. Sobotta und Herrn Prof. Borst unabhängig kontrolliert und anerkannt worden. Wir glauben daher genügend exakt gearbeitet zu haben.

$\mathrm{Zu}$ unsern Hauptversuchen stellte uns Herr Prof. Hess drei Affen zur Verfügung. Das eine Tier diente als Kontrolltier, sein Gehirnstïck wurde in der gleichen Weise gewonnen und behandelt wie das der beiden mit Botulismustoxin vergifteten Tiere.

Das erste Tier erhielt $0,0001 \mathrm{ecm}$ eines abgelagerten toxoidhaltigen Botulismustoxines subcutan unter die Bauchhaut. Nach etwa 16 Stunden zeigten sich die ersten Vergiftungserseheinungen. Das Tier, welches bis 
dahin immer anf der Stange gesessen hatte, setzte sich auf den Boden in die Ecke des Käfigs. Es wollte keine Nahrung mehr, die Pupillen reagierten noch, aber viel träger als vorher. Am andern Morgen nahm es wieder Milch zu sich, hatte aber starke Schluckbeschwerden. Die Pupillenverhältnisse blieben unverändert. Im Verlaufe des zweiten Tages hatten wir den Eindruck, dass die Mattigkeit des Tieres etwas stärker wurde. Jedoch am dritten Tage schien es sich wieder zu erhölen. Es kletterte wieder auf die Stange, aber die Pupillen blieben erweitert bei sehr träger Reaktion. Am vierten Tage erhielt das Tier von demselben Toxin 0,001 eem wieder subcutan. Schon 16 Stunden später ist es schwer krank, es sitzt in der Eeke am Boden, hält den Kopf zwischen die Beine gesenkt. Aus dem Mund fliesst beständig dicker zäher Speichel. Beim Aufrichten seines Kopfes hat das Gesicht einen eigentümlichen starren Ausdruck.

Die Pupillen sind fast maximal weit, aber reagieren immer noch, wenn auch sehr träge. 36 Stunden nach der zweiten Einspritzung hat die Schwäche des Tieres sehr zugenommen. Es liegt am Boden, versucht etwas von der gereichten Milch zu trinken, verschluckt sich fortwährend. Die Pupillen sind weit und anf Lieht absolut starr. Da es unsicher ist, ob das Tier noch weiter aushalten wird, erfolgt die Dekapitation. Der Schädel wird sofort aufgesägt, das Gehirn herausgenommen und das Vierhügelstïck in $96 \%$ Alkohol geworfen. Die ganze Prozedur hat sechs bis sieben Minuten in Anspruch genommen.

Der mikroskopische Befund ist folgender. Der Oculomotoriuskern ist in der von Bernheimer angegebenen Weise gesehnitten. Wir finden in den ersten 60 Schnitten die Kerncentra des Oculomotorius fast durchgängig im Vergleich zu der Normalserie normal. Die einzelnen Ganglienzellen zeigen scharfe Konturen, die Fortsätze sind deutlich sichtbar und oft auf eine längere Strecke zu verfolgen. Der Protoplasmaleib ist mit gleichmässigg angeordnetem und gefärbtem Tigroid ausgefüllt, der Kern grenzt sich gegen die übrige Zelle dureh eine deutliche Kermmembran ab, das Kernkörperchen ist ausgesprochen dunkler gefärbt. Im Gegensatz zu diesen Zellen finden wir solche, bei welchen der Kern nicht in der Mitte liegt, sondern randständig ist. Des weitern sind Zellen vorhanden, in denen kein Kern getroffen ist, oder ein Kern ohne Kernkörperehen vorhanden ist. Auffällig ist das Auftreten von vereinzelten Zellen, deren Protoplasma von ungleicher Färbbarkeit ist, deren Konturen weniger scharf gegen die Umgebung absetzen. Es handelt sich wohl auch hier um einzelne degenerierte Zellen, aber eine degenerierte Zellgruppe ist nirgends zu finden.

Ein wesentlich anderes Bild zeigt sich in den Schnitten von etwa 63 bis 96.

Hier ist die Situation der Kernzentren die folgende. Nach unten vom Aquaeductus Sylvii liegen seitlich von der Medianlinie die beiden Muskelkerngruppen = Seitenhauptkerne. An ihrem ventralen Ende liegt zwischen ihnen in der Medianlinie eine neue Kerngruppe, welche von Bernheimer bekanntlich als das Aceommodationszentrum angesprochen und unpaarer grosszelliger Mediankern genannt wird. Die Seitenhauptkerne gehen ohne scharfe Grenze allmählich in diese Kerngruppe über. Zwischen Seitenhauptkernen und der Medianlinie liegen dorsal nach dem Aquaeductus zu die paarigen

v. Graefe's Archiv für Ophthalmologie. LVIII. 2. 
kleinzelligen Mediankerne, welche nach Bernheimer die Zentren für den Sphincter pupillae enthalten sollen.

Die Seitenhauptkerne sind durchweg gut gefärbt, die Fortsätze der einzelnen Ganglienzellen sind zu verfolgen, die Zellkonturen sind scharf. Nur sehr vereinzelte Zellen müssen als abnorm angesehen werden.

Ausgesprochene herdförmige Degeneration dagegen findet sich in dem unparen grosszelligen Mediankern. Die Ganglienzellen sind in einem Zustand der Tigrolyse bezw. Pyknose begriffen. Bei manchen Zellen ist das Endstadium des Zerfalles erreicht. Vor allem ist auffällig, dass die Zellen nur wenig von ihrer Umgebung abstechen, dass sie viel weniger Farbstoff aufgenommen haben, wie die Zellen der Seitenhanptkerne. Die Konturen der Zellen sind sehr unscharf, manchmal ist die Grenze nur auf einer Seite etwas schärfer erkennbar. Die Zellen selbst sind wieder in einzelnen Teilen ungleichmässig gefärbt. Teilweise fehlen die Nisslsehen Granula = Tigroine, sie sind in eine staubartige Masse verwandelt, welche gleichmässig uber die ganze Zelle ausgebreitet ist. An andern Zellen finden wir die änssern Schichten enorm gefärbt, während die innern, dem Kern zunächst gelegenen gar keinen Farbstoff aufgenommen haben, ein Vorgang, den Schmauss "durch die stärkere Fürbbarkeit der Zwischensubstanz, die mit dem Tigroid verschmilzt", erklärt. Bei Zellen, an welchen die Degeneration noch weiter fortgeschritten ist, finden sich die Zellkörper verkleinert, die Konturen zackig und eckig. Die Fortsätze fehlen fast an allen Zellen ganz, oder sind nur ein kleines Stück weit zu erkennen. Wiederholt trifft man auf strukturlose Schollen, welche nur änsserst schwer gegen die Umgebung abzugrenzen sind und wohl als Reste von zu Grunde gegangenen Ganglienzellen zu bezeichnen sind. Was den Kern und das Kernkörperchen in den Zellen der degenerierten Partie anlangt, so sind die meisten Zellen ohne solche. An den Stellen, an welchen die Degeneration am ausgesprochensten ist, finden sich an einem Gesichtsfeld von 28 Zellen nur 6 kernhaltige Zellen, während in den unveränderten Seitenkernen das Verbältnis der kernhaltigen Zellen zu den kernlosen (d. h. Zellen, bei welchen der Schnitt nicht durch den Kern gelt) wesentlich anders ist. So trefen wir z. B. hier in einem Gesichtsfeld auf 18 kernhaltige 5 ohne Kern.

In den Zellen, welche noch einen Kern enthalten, zeigt derselbe, wie die Zelle selbst, hochgradige Veränderungen. Von ihm ist zumeist nur noch das Kernkörperchen erhalten. Letzteres liegt wiederholt ganz exzentrisch und stösst direkt an die Zellmembran an. In andern Zellen fehlt wieder das Kernkörperchen, die Stelle, welche den Kern enthalten sollte, zeigt eine schwach gefärbte Partie, die allmählich in die periphere stark tingierte Zone der Zelle übergeht. Vakuoläre Degeneration des Kernes können wir nicht finden. Im Gegensatz zu diesen Zellen, welche in Schrumpfung begriffen sind, finden wir auch solche, deren Leib enorm geblaht erscheint, das Zellinnere erscheint gleich den uibrigen Zellen wie mit feinem Staub bestreut.

Gegenüber diesen gewiss schweren Veränderungen im unpaaren grosszelligen Mediankern sind die Veränderungen in den kleinzelligen paarigen Mediankernen auffallend gering, wenn auch vereinzelte degenerierte Zellen zu finden sind. Durchweg erscheinen diese Kerngruppen sehr stark tingiert und lassen keine schweren Veränderungen erkennen. 
In den Schnitten nach 96 fehlt der sogenannte grosszellige Mediankern, die Seitenhauptkerne zeigen auch noch in den nächsten Schnitten änsserst vereinzelte degenerierte Zellen, ähnlich wie in den eingangs erwähnten Schnitten.

Der zweite Affe war etwas grösser und stärker als der erste. Er erhielt zunäehst $0,001 \mathrm{ccm}$ Toxin subeutan. Nach ungetähr 16 Stunden fiel es auf, dass die Pupille auf Lichteinfall träger reagierte als vorher und das schon von van Ermengem geschilderte Phänomen zeigte; sie wurden trotz Andauer des Lichtreizes wieder weit. Dieser Zustand blieb ungefähr 36 Stunden unverändert. Jetzt erhielt das Tier subeutan 0,01 ecm Toxin und schon 12 Stunden später traten schwerere Krankheitserscheinungen auf, bestehend in grosser Muskelschwäche, Diarrhöen, Speichelfluss und Schluckbeschwerden. Die Pupille zeigte noch immer die erwähnten Symptome, eine absolute Starre wurde nicht erzielt, die Reaktion war sehr träge, aber doch vorhanden, die Pupille war auch bei stundenlang anhaltender Tagesbeleuchtung über mittelweit. Die Schwäche des Tieres nahm jetzt rapide $\mathbf{z n}$, es wurde daher 30 Stunden nach der letzten Toxininjektion dekapitiert. Die Behandlung des Präparates war dieselbe wie im vorigen Fall, die Färbung geschah teils nach Nissl, teils mit Thionin. Der mikroskopische Befund war folgender: Auch hier finden wir im grossen unpaarigen Mediankern ausgesprochene Degenerationen, welche noch grösser sind als im Fall 1.

Die Degenerationen finden sich hier in den Schnitten 110-130. Dass sie 20 Schnitte später (proximaler) gelegen sind, lässt sich wohl damit erklären, dass die Schnittlinie etwas distaler gelegt worden war und das Tier etwas grösser war. In den ensten 110 Schnitten finden wir vereinzelte degenerierte Zellen in den Seitenhauptkernen, ähnlich wie in dem ersten Fall, aber ein ausgesprochener Degenerationsherd fehlt vollkommen.

In dem grosszelligen Mediankern finden wir fast alle Zellen mehr oder weniger degeneriert. Die Veränderungen sind folgende:

Die Zellen sind nur wenig tingiert. Die Konturen unscharf, so dass manche Zellen nur sehr schwer als Ganglienzellen zu erkennen sind. Viele Zellen sind zu kleinen Trümmern zerfallen, welche die verschiedensten Formen angenommen haben und teilweise ein grau-bräunliches Pigment enthalten.

Das Pigment bedeckt in stanbförmiger Anordnung die ganze Zelle oder nur einen Teil derselben, besonders die Peripherie oder einen noch erhaltenen Fortsatz.

Wiederholt finden sich sechs bis sieben Zellen nebeneinander, die vollständig zerfallen sind. Im Gegensatz zu diesen hochgradig degenerierten Zellen finden sich solche, bei welchen die Degeneration weniger ausgesprochen ist. Diese Zellen sind meist von rundlicher Gestalt, statt der Nisslsehen Granula ist der Körper mit feinen staubförmigen Einlagerungen ausgefüllt, die sich entweder gleichmässig über die ganze Zelle erstrecken, oder in den äussersten Partien besonders stark sind. Die Fortsätze fehlen überall. Bei einem Gesichtsféld, das 28 Ganglienzellen enthält, zeigen nur zwei Zellen einen feinen Fortsatz und diesen nur undeutlich.

Auch die Kernveränderungen sind hochgradig. Von den in dem er- 
wähnten Gesichtsfeld liegenden Zellen enthalten nur fünf Zellen Kerne, die übrigen haben keinen. Die Kernmembran ist nur hier und da als solche zu erkennen, meist ist rom Kern nur noch das Kernkörperchen erhalten, letzteres liegt, ohne von einer besondern Zone umschlossen zu sein, frei in der Zelle. Sehr häufig ist es randständig. Da, wo der Kern noch als solcher vorhanden ist, fehlt die Granulierung, an ihrer Stelle finden sich feinste staubförmige Einlagerungen. Auch gequollene Zellen finden sich, doch sind diese im Verhältnis zu den geschrumpften von sehr geringer Anzahl. Ausserdem trifft man noch auf kleine zackige, scharf konturierte Zellen, deren Grenzen sehr stark mit Farbstofi tingiert sind.

In den Seitenhanptkernen finden sich, wie erwähnt, nur vereinzelte degenerierte Zellen, dagegen wiederholt solche, die Pigment enthalten. Das Pigment liegt wie in den Zellen des unpaarigen Mediankernes entweder über die ganze Zelle zerstrent, oder an einer Stelle in der Peripherie und erstreckt sich oft in die Fortsätze. Die Kerne enthalten kein Pigment. $\mathrm{Ob}$ es sich nm eine Pigmentdegeneration handelt, wissen wir nicht. Es sei erwähnt, dass nach neuern Untersuchungen das Auftreten von Pigment in den Ganglienzellen kein pathologiseher Zustand der Zelle sein soll, sondern eine Alterserscheinung. In den Schnitten nach 130 lässt sich konstatieren, dass der grosszellige Mediankern aufhört, so dass nur noch die beiden Seitenhauptkerne in dem Präparat getroffen werden.

Die kleinzelligen paarigen Mediankerne, welche ungefähr in den nämlichen Schnitten wie der unpaare Mediankern getroffen sind, lassen sich noch einige Schnitte weiter verfolgen. Sie zeigen ebenso wie die Seitenhauptkerne vereinzelte Zellen, die unscharfe Konturen aufweisen und kernlos sind, ähnlich wie in dem Mediankern. Aber ausgesprochene Degenerationsherde wie im Mediankern fehlen auch in diesem Falle.

Bei der Deutung dieser anatomisch gefundenen Veränderungen im Oculomotoriuskerngebiet bei Botulismustoxinvergiftung betonen wir zunächst ausdrücklich, dass wir uns nicht für berechtigt halten, auf Grund dieser zwei erhobenen Befunde etwa in die Diskussion über das oder die Pupillencentra einzugreifen. Wohl aber wollen wir nicht verfehlen, hiermit die Fachgenossen auf die experimentelle Verwendbarkeit des Botulismustoxins bei Untersuchungen äber den Sitz jener Zentren hinzuweisen, weil wir der Überzeugung sind, dass unter Zuhilfenahme dieses von uns beschrittenen experimentellen Weges, besonders bei Untersuchung des Ruickenmarkes usw. weitere Fortschritte auf diesem Gebiete zu erzielen sein werden.

Bei unserer von uns absichtlich eng umgrenzten Versuchsanordnung kam es uns nur auf die Entscheidung der Frage an: Lassen sich die unter der Wirkung des Botulismustoxins am Auge auftretenden Frscheinungen aus einer Kernaffektion erklären?

Wir haben in Ubereinstimmung mit den Befunden von van Ermengem und Marinesco feststellen können, dass an den ver- 
Exper. Beitrag z. Frage nach d. Sitz u. Wesen d. Accommodationsparese usw. I. 307

gifteten Affen Störungen in der Pupillenreaktion auftreten. Wir haben ferner gefunden, dass deń klinischen Erscheinungen schwere Degenerationen im Oculomotoriuskerngebiet entsprechen. Und zwar waren diese Degenerationen hauptsächlich im unpaaren grosszelligen Mediankern lokalisiert, einem Kerne, der übereinstimmend als das Zentrum der Accommodation angesehen wird. Wir halten uns demgemäss für berechtigt, die These auszusprechen: Die im Verlaufe der Botulismusvergiftung auftretende Accommodationsparese hat ihren Sitz im Oculomotoriuskerngebiet und ist eine durch bestimmte Komponenten des Botulismustoxins hervorgerufene Degeneration von Ganglienzellen im Oculomotoriuskern.

Dass diese Veränderungen in den meisten nicht letal vorlaufenden Fällen beim Menschen nicht so hochgradig sein werden, wie bei den von uns vergifteten Affen, braucht wohl kaum besonders hervorgehoben zu werden. Aber auch schon Veränderungen geringern Grades reichen zweifellos hin, um die klinischen Erscheinungen erklären zu können. Gerade solche leichte Degenerationen werden sich zurückbilden können, da wir wissen, dass selbst so empfindliche Zellen wie die Ganglienzellen sehr wohl einen Teil ihrer Substanz regenerieren können.

Diese Versuche mit Botulismustoxin am Affen sind deshalb so diffizil, weil wir in diesem balteriellen Sekretionsprodukt ein furchtbares Gift besitzen, dessen einzelne Komponenten mittels partieller Absättigung noch nicht untersucht sind, und dessen Wirkung auch bei kleinen Dosen immer eine ziemlich stürmische ist. Man muss daher Tag und Nacht seine Versuchstiere kontrollieren, um jederzeit eingreifen zu können, wenn man nicht kostbares Material für einwandfreie anatomische Untersuchungen verlieren will. Etwas anders liegen die Verhältnisse wenigstens für bestimmte Tierspecies beim Diphtheriegift. Hier brauchen wir das unveränderte Gift nicht anzuwenden. $\mathrm{Ja}$, wir dürfen es für unsere Frage nicht anwenden, weil uns sonst die akute Diphtherievergiftung nicht den geringsten zuverlässigen Anhaltspunkt über den Sitz und das Wesen der in der Rekonvalescenz auftretenden Accommodationsparese gibt. Wie sich diese Verhältnisse bei dem Diphtheriegift gestalten, wo wir den Sitz der Lähmung bei dieser Krankheit zu suchen haben, darüber werden wir in einer andern Arbeit versuchen Antwort zu geben.

Für die Anregung zu diesen Untersuchungen und für die Förderung derselben sprechen wir auch an dieser Stelle unserm hochverehrten Chef, Herrn Prof. Hess, unsern ergebensten Dank aus. 
308 P. Römer u. L. Stein, Exper. Beitrag z. Frage nach dem Sitz u. Wesen usw. I.

\section{Literaturyerzeichnis.}

Literatur über Botulismus, siehe Uhth off und Groenouw. Graefe-Saemisch. 1902.

$$
\text { Speziell benutzte Arbeiten: }
$$

Schirmer, Die postdiphtheritischen Erkrankungen des Auges. Sammlung zwangloser Abhandlungen. 1896.

van Ermengem, Über einen neuen anaeroben Bacillus und seine Beziehungen zum Botulismus. Zeitschr. f. Hyg. Bd. XXVI. 1897.

Marinesco, siehe vorige Arbeit, S. 48.

Kempner und Pollak, Die Wirkung des Botulismustoxines und seines spezifischen Antitoxines auf die Nervenzellen. Deutsche med. Wochenschr. 1897. Nr. 32.

Kempner, Weiterer Beitrag zur Lehre von der Fleischvergiftung. - Das Antitoxin des Botulismus. Zeitschr. f. Hyg. Bd. XXVI. 1897.

Römer, P., Ein Beitrag zur Ätiologie des Botulismus. Centralbl. f. Bakteriologie. 1900. Bd. XXV. 